\title{
Investigations on the limitations of rapid experimental determination of stability boundaries during milling
}

\author{
Christian Brecher, Prateek Chavan*, and Alexander Epple \\ Laboratory for Machine Tools and Production Engineering (WZL) of RWTH Aachen University, Aachen, Germany
}

Received: 9 January 2017 / Accepted: 20 October 2017

\begin{abstract}
Stability boundaries in milling operations can be determined either analytically or experimentally. Since analytical determination depends on several assumptions and approximations, experimental methods offer a realistic alternative. This however, requires numerous milling trials with different process parameters the results of which are then illustrated in a stability lobe diagram. Although the methods for rapid experimental determination of stability boundaries such as continuously increasing depth of cut and continuously increasing cutting speed have been suggested in literature, they have not been adequately evaluated for their accuracy and limitations. In the current paper, the method of continuously increasing depth of cut is analysed. For this, a Poincaré-section based real time chatter detection algorithm was firstly calibrated. Subsequently, this algorithm was run along with milling trials with continuously increasing depth of cut by inclining the work piece. The influence of the inclination angle on the stability boundary could then be analysed.
\end{abstract}

Keywords: Chatter detection / Poincaré / stability lobes / increasing cutting depth

\section{Introduction}

The milling process is a key technology for manufacturing complex components for the mould and die, aeronautical, process and automotive industries. High productivity and financial viability of a milling operation is achieved by having a maximum material removal rate (MMR). MMR is in turn limited by the maximum achievable depth of cut without instability for the particular combination of tool, work piece, machine structure, machine position and process parameters. Besides optimizing the process parameters, current research also focuses on optimizing tool geometry. In this context, Comak [1] analyses the chatter stability of variable pitch and helix tools and develops a method to calculate the optimum tool geometry for increased stability during cutting.

Instability during milling arises mainly due to selfexcited vibrations or "chatter". Prediction of instability due to chatter has been the subject of extensive research for the past decades. The instability boundaries are often plotted in a so-called stability lobe diagram (SLD). These boundaries can be determined either empirically or through simulation. Several analytical [2], numerical [3] and time domain [4] simulations have already been proposed in literature. However, an accurate simulative

\footnotetext{
* e-mail: p.chavan@wzl.rwth-aachen.de
}

prediction is often not achievable without making several assumptions and extensive measurements of machine dynamics, cutting stiffness's as well as the calculation of constant and time variant cutting force coefficients. These measurements and calculations are time consuming and effortful. For example in [2], Altintas and Budak propose an analytical description of the directional dynamic cutting force coefficients. The implementation of this method requires the calculation of these coefficients in advance along with the prior measurement of the transfer function matrix at the tool tip-workpiece contact zone. As this requires a lot of time, effort and expertise to implement for each combination of tool and workpiece, such simulative methods have not found application in the industry.

On the other hand, the empirical determination of stability boundaries is usually more accurate and reliable than an analytical prediction. Again, this experimental determination is valid only for the given combination of tool, work piece, machine and process. Despite higher accuracy, experimental investigations of stability boundaries are still not widely implemented in industrial applications. This is because of the large number of cutting experiments that are required to create a SLD.

In order to reduce the time and effort associated with these experiments, Quintana et al. [5] suggest the use of continuously increasing depth of cut starting from zero millimetres up to the critical depth of cut for a constant 
spindle speed instead of performing an experiment for each combination of process parameters. A continuously increasing depth of cut was realised by having the workpiece inclined by a certain angle and keeping the axial position of the tool constant. For the online detection of the onset of chatter, a fast Fourier transform (FFT) of the audio signal obtained during the milling of the slots was utilized. The presence of a high amplitude at the chatter frequency indicated the onset of chatter. The assumption for the detection is that regenerative effect causes the instability. Weingaertner et al. [6] showed that in case of finish milling, forced vibration could also cause instability, which cannot be detected by observing the vibration amplitude at chatter frequency in the FFT. A further disadvantage of the frequency domain method is the inherent time delay associated with the higher resolutions of the FFT.

In [7] an alternative method for rapid determination of SLDs was proposed. This involved a ramp up of the spindle speed and the instability detection using a statistical chatter indicator. The advantage is that no previous measurement of the machine is necessary and that detection occurs on-line during milling operation. Recently, Grossi et al. performed a similar ramp-up test while keeping the feed per tooth constant [8]. Additionally, for chatter identification, an order analysis technique in the frequency domain was utilized. Although this method could be implemented on-line, it suffers from the disadvantages associated with frequency domain methods such as low frequency resolution, time delay and reliable detection only after a chatter occurrence.

An on-line time-domain instability detection method was proposed in [9] based on the Poincaré approach suggested in [10]. This method is based on the fact that the position signals near the tool centre point (TCP), obtained by double integration, follow the same trajectory on the Poincaré map for a stable process. For an unstable process, however, the trajectory is chaotic and non-consistent. Hence, the transition from stable to unstable can be recognized by observing the Poincaré-section, a lower dimensional subspace of the Poincaré map. In this case, a Poincaré-section includes the position of the TCP for different revolutions but at just one specific angular position of the spindle. The result is a scatterplot. In [10] this chatter detection method was combined with milling tests with continuously varying cutting depths.

As seen from the available literature, the approaches of either continuously increasing depth of cut or spindle speed have been combined with a frequency or time domain based instability detection algorithm for rapidly determining SLDs. However, the following two aspects remain unexplored:

- An extensive validation of the chatter detection method and the calculated critical depth of cut.

- The influence of the process parameters on the stability boundary detected by rapid methods of determining SLDs. For example, the influence of spindle acceleration on stability in case of continuously increasing spindle speed. Alternatively, influence of workpiece inclination angle in case of continuously increasing cutting depths.
In this paper, the Poincaré-section based time domain method introduced in [9-11] was implemented, extensively validated and calibrated (Sects. 2 and 3). After that, a preliminary investigation of the influence of varying the inclination angle on the detection of the stability boundary (Sect. 4) was performed.

\section{Time domain detection of chatter vibrations}

The Poincaré-section method is based on two dimensional vibration, i.e. vibration in a plane. In order to detect instability, the displacement of the tool over a certain number or revolutions must be analysed in this plane. The plot of the two-dimensional displacement $(X-$ and $Y$ coordinate) of the tool is called the trajectory and is equivalent to a Poincaré-map. The angular position of the tool must be known at all points of the trajectory (Fig. 1a). The points on the trajectory corresponding to a particular tool angle can be defined as the Poincaré-section. Considering the trajectory over several revolutions of a stable process, the trajectory points at the same angular position will lie close to one another forming a cluster of bisection points (blue points in Fig. 1a). For an unstable process, this trajectory becomes chaotic and the bisection points do not lie close to one another as seen in Figure 1a. The stability of the process can hence be analysed by considering the state of the trajectory. In this paper the ratio of the standard deviations of the bisection points, $s_{B x}$ and $s_{B y}$, to that of the complete trajectory, $s_{T x}$ and $s_{T y}$, are used as the chatter indicator (CI).

$$
\mathrm{CI}=\frac{s_{B x} \cdot s_{B y}}{s_{T x} \cdot s_{T y}} .
$$

For a stable process, this ratio remains small and increases due to instability. Chatter is detected by using specific criteria over time as shown in Figure 1b. Therefore, the estimation of stability is based on current and previous values of the CI. For example, the gradual increase of amplitude of vibration leading to chatter can be detected by the positive trend criterion. Therefore, a defined trend factor $T$ is a boundary value to identify critical trends in the CI. The sudden increase of vibration amplitude can also be identified as instability by observing the jump in amplitude of the current chatter indicator with that of the mean of the previous five chatter indicators. Here the leap factor $S$ influences the result. As a safety measure, an absolute limit $G$ on the vibration amplitude is set for avoiding damage to tool and machine. The values of factors $T, S$ and $G$ can be calibrated for a workpiece-tool combination.Since the tool tip vibration cannot be measured directly, an acceleration sensor is set on the non-rotating part of the spindle. The vibration plane is chosen as the horizontal plane parallel to the milled surface. In addition, the chatter indicator for the measurement signals is calculated every $0.1 \mathrm{~s}$. This approach is implemented as LABVIEW routine and can be run online with the milling trials. 


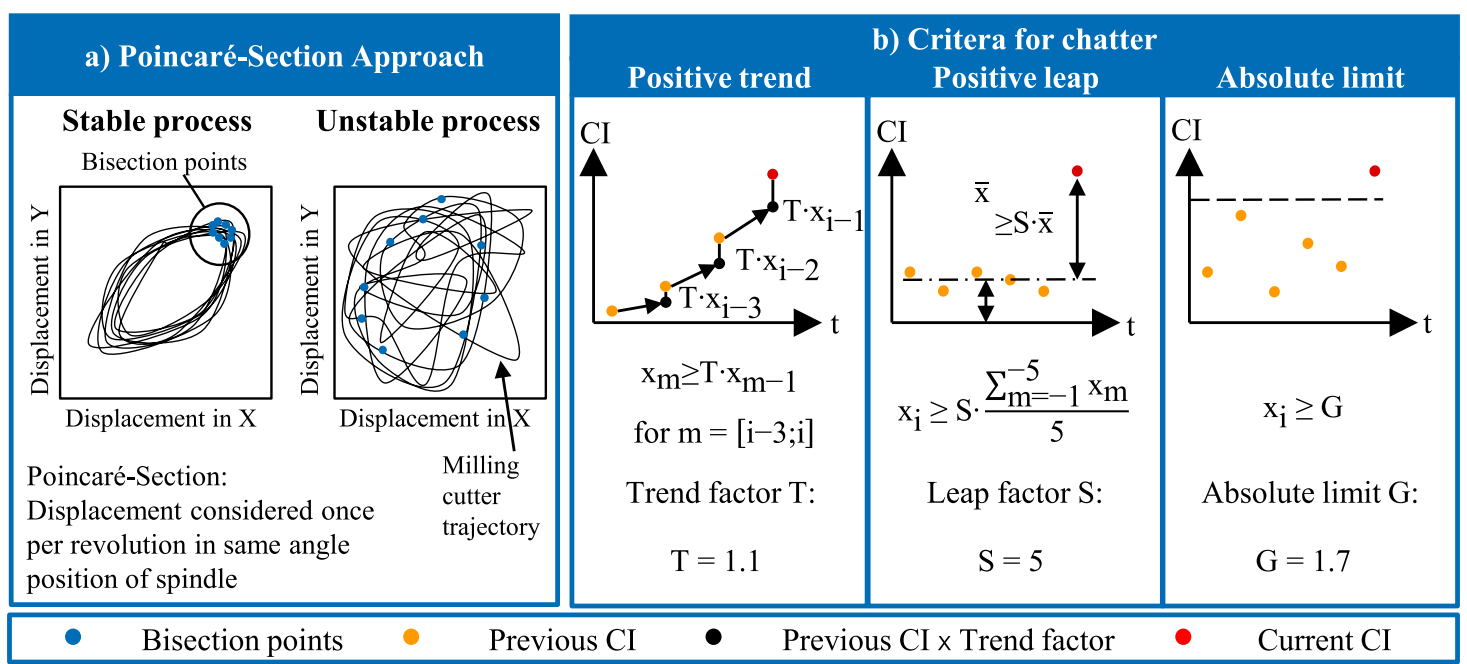

Fig. 1. (a) Trajectory of Poincaré-points for stable and unstable process and (b) criteria for chatter.

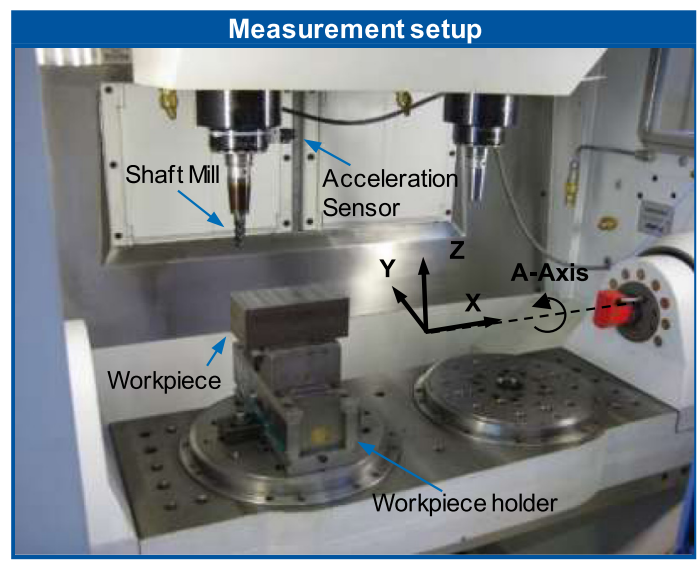

Fig. 2. Experimental setup for slot milling trials.

\section{Experimental setup}

Slot milling operations were conducted on a prototype double-spindle machining centre. The work piece used it was medium carbon steel of grade $\mathrm{C} 45$. The dimensions of the work piece were $150 \times 80 \times 60 \mathrm{~mm}$. The tool used was a 4-toothed carbide shaft mill H3 Series of diameter $16 \mathrm{~mm}$ and constant helix angle from Walter tools. The feed rate per tooth was kept constant at $0.01 \mathrm{~mm} /$ tooth. The block was cut along it's width in the $Y$ direction according to the machine coordinate system (Fig. 2). The length of cut was kept low to minimize the influence of position dependent machine dynamics. The spindle speed was varied between 2000 and $4000 \mathrm{rpm}$ in steps of $250 \mathrm{rpm}$, which corresponds to nine slot-milling operation with full immersion. The continuous increase in cutting depth was achieved by having an inclination angle of the work piece by rotating the $A$-axis correspondingly. For the investigations, this angle could be varied. Starting at a value of zero cutting depth was increased continuously until chattering was detected. Acceleration was measured at the spindle near the tool as shown in Figure 2. The angular position of the spindle was obtained by extracting the interpolator signals from the control system. The milling path was kept relatively short for minimizing the influence of position dependent dynamics of the machine.

\section{Calibration and validation of the detection method}

Due to the increasing depth of cut, there is a transition from a stable to an unstable process on exceeding the critical depth of cut. Two types of transitions to instability are observed. One where the transition is distinct and abrupt and the other where it is gradual and ill defined. The chatter recognition method was analysed and validated for each of these cases.

\subsection{Distinct transition}

In case of an abrupt transition, the stability boundary can be identified easily by observing the surface quality of the milled surface or the acceleration signal (Fig. 3). This allows an excellent benchmark for calibrating and validating the chatter detection algorithm. Out of the nine experiments at different spindle speeds, four showed distinct transitions and five showed a gradual transition (Tab. 1). In order to calibrate the detection algorithm, measurement data of one of the cuts showing distinct transition was utilized. In this case, measured data from Trial 1 was used and the parameters $T$ and $G$ were adjusted. These values were then kept unchanged for the other trials. The chatter detection algorithm was executed real time during the milling operation. The left side of Figure 3 shows, as an example, the comparison of the milled surface of Trial 6 , the corresponding acceleration signal, the progression of the chatter indicator for the $X Y$ plane over time and the metrological detected surface roughness in terms of $R z$ values. The surface profile shows a clear change in roughness at the chatter-affected surface. Before the onset of chatter, the surface shows lower $R z$ values. 


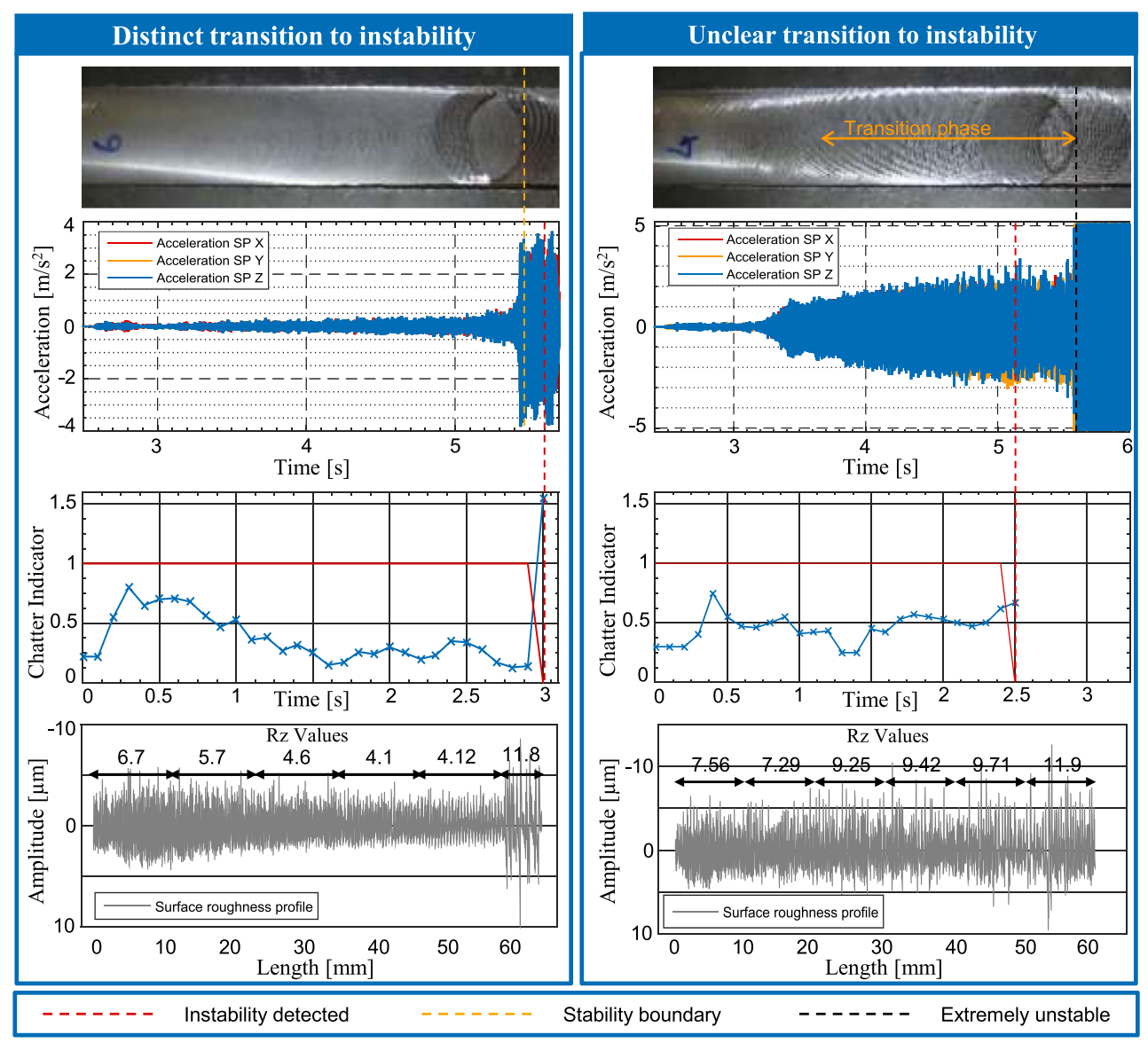

Fig. 3. Visualisation of acceleration and the resulting CI for distinct and gradual transition.

The surveillance for instability is triggered only after the tooth penetration with work piece is detected. This is the reason why the time scale for the chatter indicator begins at $0 \mathrm{~s}$. As can be observed, upon the onset of chatter, the chatter indicator also shows a sudden increase in its value. This increase is then recognized as a positive leap and the software notifies the onset of instability to the machine user. In case of distinct transition, the detected and measured stability boundaries show an excellent correlation. Stability boundary was measured by identifying the depth of cut at the point of chatter as shown in Figure 3. Since the detection of instability takes place every $0.1 \mathrm{~s}$, there is a time delay between the occurrence and detection of chatter. This can be observed in Figure 3 (left).

\subsection{Gradual transition}

In case of a gradual transition, the onset of chatter is unclear and is subjective due to the lack of an abrupt change of the CI. For this reason, such transitions are not suitable for the validation of the chatter detection algorithm. This can also be seen in the surface roughness profile (Fig. 3, right). For analysis, the machined length was divided into six parts of $10 \mathrm{~mm}$ each and the $R z$ values for each strip was measured. A gradual increase in surface roughness can be observed without any clear boundary. The chatter detection algorithm can, however, be calibrat- ed such that chatter criteria corresponds to the subjective assessment of the machine operator. The gradual increase in vibration amplitude can be detected as chatter only with the help of the "positive trend"-criterion for instability. This criterion can be calibrated by varying the trend factor $T$ or by varying the number of previous chatter indicators $m$ to be considered for identification of trend.

A conventional SLD was obtained for spindle speeds between 2000 and $4000 \mathrm{rpm}$ by performing 38 cutting trials at discrete spindle speeds (steps of $250 \mathrm{rpm}$ ) and depths of cut. At those spindle speeds where the stability boundary was not distinct, the decision for instability was established subjectively by referring to the extent of chatter marking and the machine operator experience. Based on this assessment, the parameters for the positive trend criteria were adjusted for one of the gradual transitions and then kept constant for all other trials.

Table 1 shows the comparison between the critical depth of cut detected by the calibrated algorithm and the subjectively identified critical depth of cut. The critical depth of cut obtained by milling trials with constant depth of cut at constant spindle speed are considered as the true stability boundary. It can be seen that, except for Trial 8 , the critical cutting depth calculated by the chatter detection algorithm corresponds well with the measured critical depth. Furthermore, due to the subjective nature of the benchmark, the calculated critical depth showed a larger error for gradual transition. 
Table 1. Validation and calibration of the detection algorithm.

\begin{tabular}{llllll}
\hline Trial no. & $\begin{array}{l}\text { Spindle speed } \\
(1 / \mathrm{min})\end{array}$ & $\begin{array}{l}\text { Calculated } \\
\text { critical cutting } \\
\text { depth }(\mathrm{mm})\end{array}$ & $\begin{array}{l}\text { Measured } \\
\text { critical cutting } \\
\text { depth }(\mathrm{mm})\end{array}$ & Error $(\%)$ & $\begin{array}{l}\text { Average and } \\
\text { standard deviation } \\
(\%)\end{array}$ \\
\hline $\begin{array}{l}\text { Distinct transition } \\
1\end{array}$ & & & & $5.62 ; 4.5$ \\
2 & 2500 & 0.77 & 0.76 & 1.30 & \\
3 & 2750 & 0.57 & 0.52 & 8.80 & \\
6 & 3000 & 0.71 & 0.79 & 11.30 & \\
& 3750 & 0.83 & 0.82 & & \\
Gradual transition & & & & \\
4 & 3250 & & & & \\
5 & 3500 & 0.43 & 0.41 & -7.70 & \\
7 & 4000 & 0.52 & 0.56 & 0.00 & \\
8 & 2000 & 0.48 & 0.48 & -39.30 & \\
9 & 2250 & 0.56 & 0.78 & 7.60 & \\
\hline
\end{tabular}

Table 2. Summary of milling trials at different workpiece inclination angles (distinct transitions).

\begin{tabular}{|c|c|c|c|c|c|c|c|c|}
\hline \multirow{3}{*}{$\begin{array}{l}\text { Spindle } \\
\text { speed } \\
(1 / \mathrm{min})\end{array}$} & \multirow{3}{*}{$\begin{array}{l}\text { Conventionally } \\
\text { determined } \\
\text { stability } \\
\text { boundary }(\mathrm{mm})\end{array}$} & \multicolumn{7}{|c|}{ Continuously increasing depth of cut } \\
\hline & & \multicolumn{2}{|c|}{$\alpha=0.5^{\circ}$} & \multicolumn{2}{|c|}{$\alpha=0.75^{\circ}$} & \multicolumn{2}{|c|}{$\alpha=1^{\circ}$} & \multirow[t]{2}{*}{ Mean error $(\%)$} \\
\hline & & $\begin{array}{l}\text { Detected } \\
\text { critical } \\
\text { depth } \\
(\mathrm{mm})\end{array}$ & $\begin{array}{l}\text { Error } \\
(\%)\end{array}$ & $\begin{array}{l}\text { Detected } \\
\text { critical } \\
\text { depth } \\
(\mathrm{mm})\end{array}$ & Error $(\%)$ & $\begin{array}{l}\text { Detected } \\
\text { critical } \\
\text { depth } \\
(\mathrm{mm})\end{array}$ & Error $(\%)$ & \\
\hline 2000 & 0.78 & 0.56 & 28.2 & 0.85 & 9.0 & 0.67 & 12.9 & 16.71 \\
\hline 2250 & 0.61 & 0.66 & 8.2 & 0.84 & 37.7 & 0.68 & 8.3 & 18.08 \\
\hline 2500 & 0.76 & 0.77 & 1.3 & 0.76 & 0.0 & 0.88 & 15.8 & 5.70 \\
\hline 2750 & 0.61 & 0.57 & 6.6 & 0.62 & 1.6 & 0.67 & 9.7 & 5.96 \\
\hline 3000 & 0.76 & 0.71 & 6.6 & 0.73 & 3.9 & 0.8 & 5.5 & 5.34 \\
\hline 3250 & 0.41 & 0.43 & 4.9 & 0.68 & 65.9 & 0.75 & 50.0 & 40.24 \\
\hline 3500 & 0.56 & 0.52 & 7.1 & 0.48 & 14.3 & 0.57 & 2.1 & 7.84 \\
\hline 3750 & 0.78 & 0.83 & 6.3 & 0.78 & 0.0 & 0.82 & 5.1 & 3.80 \\
\hline 4000 & 0.48 & 0.48 & 0 & 0.45 & 6.25 & 0.32 & 35.6 & 13.94 \\
\hline
\end{tabular}

\section{Results}

Subsequently, milling trials were conducted at different spindle speeds and workpiece inclination angles $\alpha$. In Table 2 , the critical depths of cut identified by continuously increasing the depth of cut combined with the online chattering-algorithm (Poincaré) for different $\alpha$ s are compared with those identified through the conventional method. For spindle speeds 2500, 2750,3000 and $3750 \mathrm{rpm}$, where the stability has distinct transition (marked as bold in Tab. 2), the deviation of stability boundary is significantly lower compared to those where the stability boundary is unclear. This indicates that in the case where stability boundaries are established subjectively, the detection algorithm could not consistently determine the same stability boundary. Therefore, an objective criterion is necessary to enable the accurate detection of chatter boundaries.

The influence of the inclination angle was analysed only for distinct transition spindle speeds (highlighted in blue in Fig. 4). As discussed before, the stability boundary obtained by the conventional method is considered as the true critical depth. Irrespective of the inclination angle, the detected boundaries for distinct transition spindle speeds show a smaller deviation from the true boundary as compared with the other speeds. The inclination angles of 


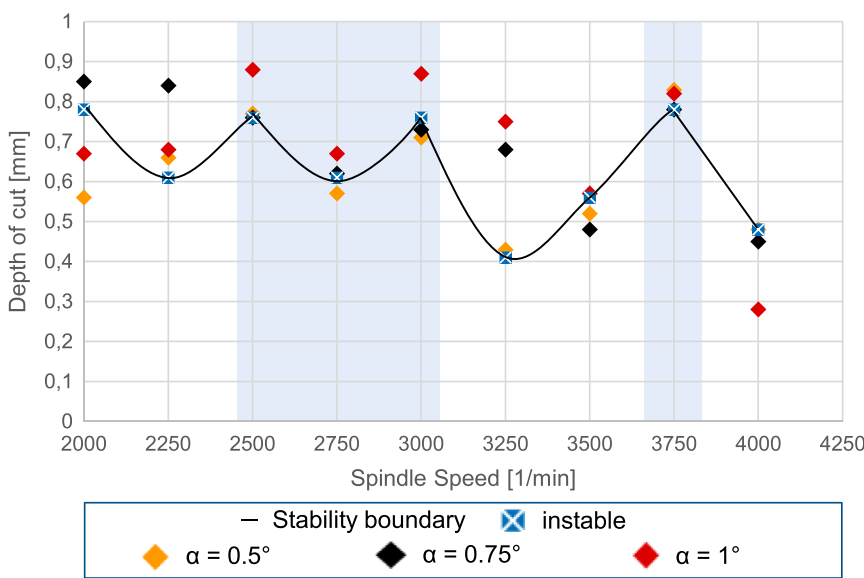

Fig. 4. Conventional SLD with detected stability boundaries for different inclination angles.

$0.5^{\circ}$ and $0.75^{\circ}$ show an almost identical boundary as the true boundary. With an incident angle of $1^{\circ}$, the process seems to show a higher critical depth of cut. Further experiments and trials need to be conducted to explain this behaviour sufficiently. However, two possible factors could be: (1) a marginally delayed chatter detection results in a larger error of critical depth of cut for higher inclination angles; (2) at lower inclination angles the dynamic process force increases gradually and the machine tool structure oscillates longer at the same depth of cut and could trigger the onset of chatter. This is not the case for higher inclination angles.

\section{Conclusion}

A time domain chatter detection algorithm based on the Poincaré-section method was implemented, validated and calibrated such that instabilities could be detected for continuously increasing depths of cut. For validating the accuracy of the algorithm, those spindle speeds, which show a distinct transition to instability, must be chosen. For those, which do not, an objective chatter criterion or a modified detection criterion must be established, for example based on process forces, to enable accurate identification of stability boundaries. It was also observed that higher inclination angles of the work piece led to higher stability boundaries. To avoid inaccuracies in the detection, the inclination angle should be kept to a minimum.

Acknowledgement. The authors wish to gratefully acknowledge the support of the German Research Foundation (Deutsche Forschungsgemeinschaft, DFG). This work was funded as part of the DFG Project "Efficient determination of SLDs" (Project Number BR 2905/73-1).

\section{References}

[1] A. Comak, E. Budak, Modeling dynamics and stability of variable pitch and helix milling tools for development of a design method to maximize chatter stability, Precis. Eng. 47 (2017) 459-468

[2] Y. Altintas, E. Budak, Analytical prediction of stability lobes in milling, Ann. CIRP 44 (1995) 357-362

[3] R. Sridhar, R.E. Hohn, G.W. Long, A stability algorithm for the general milling process, Trans. ASME J. Eng. Ind. 90 (1968) 330-334

[4] S. Smith, J. Tlusty, Update on high speed milling dynamics, Trans. ASME J. Eng. Ind. 112 (1990) 142-149

[5] G. Quintana, J. Ciurana, D. Teixidor, A new experimental methodology for identification of stability lobes diagram in milling operations, Mach. Tools Manuf. 48 (2008) 1637-1645

[6] W.L. Weingaertner, R.B. Schroeter, M.L. Polli, J. de Oliveira Gomes, Evaluation of high-speed end-milling dynamic stability through audio signal measurements, Mater. Process. Technol. 179 (2006) 133-138

[7] F. Ismail, E. Soliman, A new method for the identification of stability lobes in machining, Mach. Tools Manuf. 37 (1997) $763-774$

[8] N. Grossi, A. Scippa, L. Sallese, R. Sato, G. Campatelli, Spindle speed ramp-up test: a novel experimental approach for chatter stability detection, Mach. Tools Manuf. 89 (2015) 221-230

[9] C. Brecher, A. Epple, M. Fey, Optimale Prozessparameter effizient ermitteln, wt Werkstatttechnik online 104 (2014) 260-265

[10] T. Surmann, Geometrisch-physikalische Simulation der Prozessdynamik für das fünfachsige Fräsen von Freiformflächen, Thesis, Dortmund University, 2005

[11] C. Brecher, C. Kiesewetter, A. Epple, M. Fey, Automatisierte Erstellung von Stabilitätskarten für Fräsbearbeitungen, ZWF 110 (2015) 191-195

Cite this article as: C. Brecher, P. Chavan, A. Epple, Investigations on the limitations of rapid experimental determination of stability boundaries during milling, Mechanics \& Industry 18, 608 (2017) 